\title{
Reconditionnement énonciatif et reconfiguration discursive dans le discours de la mise en scène de soi : l'exemple des autographies de JJ Rousseau.
}

\author{
Pascale Delormas \\ Paris $12 /$ Ceditec \\ pascale.delormas@orange.fr
}

\section{Introduction}

Les notions de «reconditionnement énonciatif » et de «reconfiguration discursive » renvoient à l'acte volontaire de reformulation d'un même propos orienté vers un objectif communicationnel, le premier terme entretenant une relation de cause à effet avec le second. Elles permettent de rendre compte sinon d'une stratégie - ce terme a le défaut de placer l'auteur en position d'extériorité par rapport à son acte alors qu'il en est l'émanation même - du moins de la participation à l'interdiscours.

Ces notions s'avèrent particulièrement efficaces dans l'analyse des «autographies »- que nous distinguons des simples écrits de soi qui n'ont pas la prétention de faire œuvre ${ }^{1}$. Dès lors que les autographies sont envisagées comme des discours de mise en scène de soi, elles réclament une approche fort éloignée d'une conception romantique de l'expression de l'intime. Les aborder comme des « configurations » rend justice à la notion développée par P. Ricœur (1983) dans le cadre pragmatique de l'interaction verbale. Il s'agit de décrire les enjeux de l'autographie et de prendre en considération l'illusion biographique entretenue par les autographies comme le résultat d'un « ensemble d'instructions que le lecteur ou le public exécutent de façon passive ou créatrice $»^{2}$ car «[La] visée d'un référent du discours est rigoureusement contemporaine de son caractère d'événement et de son fonctionnement dialogal. ${ }^{3}$ » L'acte de configuration étant autant un acte de production-schématisation que de lectureinterprétation, l'analyste de discours cherche à débusquer dans l'énoncé les procédés qui forcent la conviction.

Je me propose d'approcher les « autographies » de Rousseau comme autant de configurations discursives d'un même propos liées à un reconditionnement énonciatif. Contrairement à la conception romantique de l'expression d'un moi polymorphe, le caractère répétitif des Confessions, des Dialogues et des Rêveries de Rousseau indique clairement la volonté d'un positionnement dans le champ discursif. Ils sont le témoignage d'une identité énonciative ouverte qui se maintient à travers l'interdiscours par un travail incessant de reconfiguration. Pour mener l'analyse de leur spécificité discursive, trois niveaux d'énonciation étroitement liés sont considérés : le dialogisme interdiscursif qui mobilise des références génériques, les marques linguistiques de l'énonciation et enfin les modalités de prise en charge du discours rapporté.

\section{Les références génériques}

Le discours autographique est une catégorie discursive transverse à plusieurs genres qui fait l'objet d'un cadrage auctorial. Chaque autographie résulte du couplage d'une étiquette générique et d'une réalité communicationnelle. La scène d'énonciation construite par le texte relève de la «captation» de catégories génériques routinières contemporaines et d'œuvres qui appartiennent à la culture commune pour «transférer sur un discours réinvestisseur l'autorité attachée au texte ou au genre source ${ }^{4}$ ». Les scénographies des discours autographiques de Rousseau sont à la croisée de virtualités liées à plusieurs genres de référence, comme la confession (scène générique tirée de l'univers religieux) et la méditation (scène générique dans la sphère privée) et aux hypergenres du dialogue (univers mondain et philosophique) et du soliloque. 


\subsection{Les Confessions}

Le terme de «Confessions » n'est pas un simple étiquetage générique, c’est aussi un titre. A la faveur de l'engagement de sincérité que la pratique de la confession suppose, Rousseau capte une catégorie routinière de son temps et se positionne explicitement dans un interdiscours pour développer un projet personnel. Il s'agit de convaincre un public de la valeur de son œuvre et de sa valeur propre en référence à la tradition et contre elle.

Par le titre de «Confessions", Rousseau se réfère à une activité verbale interactive de l'Eglise catholique : le genre de la confession renvoie à la révélation d'actes répréhensibles, dans la perspective de l'effacement des fautes; elle a lieu à huis clos et l'auditeur, le prêtre, est médiateur entre le locuteur et Dieu. Cependant, la référence lourde de sens à un rite que les protestants n'observent pas est modérée par la déclaration inaugurale : l'interlocution directe avec le « souverain juge » renvoie à sa critique virulente de l'Eglise catholique qui lui a fait abjurer sa foi.

Que la trompette du jugement dernier sonne quand elle voudra, je viendrai, ce livre à la main, me présenter devant le souverain juge. Je dirai hautement : Voilà ce que j'ai fait, ce que j'ai pensé, ce que je fus. [...] Etre éternel rassemble autour de moi l'innombrable foule de mes semblables [...] que chacun d'eux découvre à son tour son cour au pied de ton trône avec la même sincérité $[\ldots]^{5}$

Quant à la tradition de la révision de vie, Rousseau est loin d'être le premier à prétendre faire œuvre de vérité dans un auto-examen. Depuis la Grèce antique il est d'usage d'écrire des textes à son propre propos; les bios héllenistiques et les commentari de vita sua très nombreux; les bios socratiques sont considérés comme un art d'accouchement de la connaissance à travers la connaissance de soi. Une des premières formes d'enseignement chez les chrétiens semble avoir été celle du témoignage (la vie des saints) et a pris plus tard d'autres formes comme la «révision de vie ». Au XIVe siècle, l'écriture du domaine privé sera le moyen d'entrer dans le débat qui oppose les églises. Plus tard, «au travers d'une masse d'autographies que les réactions piétistes et méthodistes suscitent dans l'Europe des XVIIe et XVIIIe siècles, se développe un christianisme de la première personne et se constituent les modèles d'une rhétorique du moi qui rassemblent dans une même pratique introspection et spiritualité, investigation et $\operatorname{confession}^{6} \gg$.

\subsection{Les Dialogues}

Nous envisageons les Dialogues de Rousseau comme la poursuite de son entreprise de mise en scène de soi engagée avec les Confessions. La problématique est la même, les commentaires n'ont pas changé, les objectifs poursuivis non plus. Les Dialogues, comme les Confessions, réfèrent à la fois à un environnement générique traditionnel et empruntent aux pratiques discursives du temps. En revanche, les choix énonciatifs diffèrent profondément. Le récit des Confessions propose une mise en place monologale de son procès. Dans les Dialogues, pour exposer une nouvelle fois sa défense, Rousseau procède à un recadrage dans une forme dialogale.

La scénographie des Dialogues renvoie à une pratique conversationnelle et dramatique du dialogue par son étiquetage : le titre des Dialogues renvoie à l'hypergenre du dialogue, c'est-à-dire au formatage peu contraignant de la pratique conversationnelle (en l'occurrence, une alternance de tours de parole) dans laquelle les auteurs inscrivent des dispositifs d'énonciation si variés que l'on ne peut pas le considérer comme un genre.

Le premier titre, Rousseau juge de Jean-Jacques, Dialogues, manifeste un double emprunt, au genre routinier des mémoires juridiques et à la classe généalogique ${ }^{7}$ du dialogue philosophique. Pratique sociale de référence, le genre routinier du «mémoire juridique » recouvre une réalité fort commune au XVIIIe siècle. À l'époque où Rousseau écrit ses Dialogues, l'opinion manifeste un véritable engouement pour les affaires judiciaires ${ }^{8}$. Destinés à pallier l'arbitraire et le secret, ils sont publiés par les avocats et lus avec intérêt par le public qui tend à se représenter lui-même comme tribunal supérieur de la Nation. Ils constituent le seul intermédiaire réel entre la sphère de la justice et celle des réalités extérieures de la 
société civile. Le mémoire judiciaire courant est rédigé à la première personne ; il recourt délibérément aux procédés d'accréditation de vraisemblance qui sont ceux de la fiction romanesque, et il peut mettre en scène et faire parler tous les acteurs de l'affaire, acteurs judiciaires compris.

\begin{abstract}
Rousseau : [...] Mais, Monsieur, à ce compte, cet homme chargé de tant de crimes n'a donc jamais été convaincu d'aucun?

Le Français : Eh non vraiment. C'est encore un acte de l'extrême bonté dont on use à son égard de lui épargner la honte d'être confondu. Sur tant d'invincibles preuves n'est-il pas complètement jugé sans qu'il soit besoin de l'entendre. Où règne l'évidence du délit la conviction du coupable n'est-elle pas superflue ? Elle ne ferait pour lui qu'une peine de plus. En lui ôtant l'inutile liberté de se défendre on ne fait que lui ôter celle de mentir et de calomnier. ${ }^{9}$
\end{abstract}

La classe généalogique du dialogue philosophique est également convoquée : ainsi, le titre Dialogues renvoie-t-il au dialogue platonicien. La vogue des «entretiens » ou les «dialogues galants » du siècle précédent, qui visaient à mettre un savoir, supposé austère, à la portée d'honnêtes gens, est délaissée pour que s'instaure ce mode contestataire qu'est le dialogue philosophique. Le mode d'organisation textuelle est adapté à son objectif : caractère didactique, argument et enjeu sont explicités dans le paratexte «Du sujet et de la forme de cet écrit » :

La forme du dialogue m'ayant paru la plus propre à discuter le pour et le contre, je l'ai choisie pour cette raison. ${ }^{10}$

\title{
2.3 Les Rêveries du promeneur solitaire
}

Rousseau a procédé pour les Rêveries à un «formatage», qui se justifie, comme le dialogue, pour des raisons fonctionnelles. La promenade, comme hypergenre, offre les mêmes possibilités que celui de la conversation : les Rêveries sont un recueil dont l'organisation textuelle mime le vagabondage.

La scénographie des Rêveries s'inspire de la classe généalogique de la promenade, mise en scène d'un espace de sociabilité marqué par la pratique de la conversation. Propice à la méditation, la promenade a les faveurs des philosophes tentés par cette forme souple qui permet la mise en scène du dialogue auquel elle sert de fond : dans les dialogues platoniciens, le maitre et l'élève déambulent de conserve ${ }^{11}$. Revisitée par de nombreux auteurs du XVIIe et du XVIIIe siècle, la promenade donne à la philosophie une nouvelle dimension : elle devient pratique sociale.

Mais Rousseau se désolidarise des pratiques mondaines par une sorte de contestation de la promenade : le titre définit un pacte de lecture singulier; le promeneur est solitaire. L'entretien avec soi-même suppose l'exclusion du mondain et doit ménager une interaction fictive. La définition programmatique et la thématique présentée dans les Rêveries nous amènent à considérer l'investissement de Rousseau dans l'hypergenre de la méditation philosophique comme l'action d'un « vrai philosophe », qui choisit la vie dans le désert ${ }^{12}$. Cependant, la méditation elle-même est contestée par le commentaire désinvolte du promeneur lorsqu'il manifeste son manque de goût cet exercice et sa préférence pour la rêverie :

[...] quelquefois mes rêveries finissent par la méditation, mais plus souvent mes méditations finissent par la rêverie, et durant ces égarements, mon âme plane et erre dans l'univers sur les ailes de l'imagination dans des extases qui passent toute autre jouissance. ${ }^{13}$

Les termes du dialogisme interdiscursif que nous venons de passer rapidement en revue montrent comment la captation des genres et l'adoption de pratiques discursives permet un positionnement dans la communauté discursive. L'approche linguistique de l'énonciation confirme notre analyse des différents choix génériques comme tentatives de singularisation dans la communauté discursive. 


\section{Approche linguistique de l'énonciation}

Les dispositifs énonciatifs qui articulent les différents types de narration dans les Confessions et les Rêveries introduisent par l'artifice d'un je intermédiaire, sujet de mémoire, un tiers, là où Rousseau dit l'avoir exclu de son champ. Les Dialogues s'appuient sur un autre procédé, le dialogue entre deux personnages. Les Rêveries semblent hériter les procédés des deux œuvres qui les précèdent, puisqu'elles prétendent aussi résulter d'un dialogue avec soi-même.

\subsection{Introduction d'un Je de mémoire}

Indépendamment du paratexte et du pacte de lecture qu'implique la référence aux genres existants, le dispositif énonciatif des Confessions construit une scénographie qui joue des modes d'énonciation et de la présence plus ou moins explicite du locuteur dans des séquences narratives de types variés. Toute œuvre mettant en scène un énonciateur distinct de l'auteur, c'est sur la base de cet être de discours que se construit la scène d'énonciation qui s'affirme dans le texte comme l'instance du locuteur. Les textes autographiques posent des problèmes redoutables à qui veut distinguer les je en présence. Cela tient au fait que le locuteur, le narrateur et le personnage sont à identifier comme les mêmes, conformément sans doute à notre conception anthropologique d'un moi plein et au fait que le texte autographique est «mise en récit » ou « mise en intrigue » du moi qui témoigne d'une temporalité vécue par le locuteur.

Le caractère particulier des Confessions se manifeste dans l'alternance des mises en scène des divers je et par l'enchâssement d'énoncés hiérarchisés. Rousseau, en prétendant faire appel à ses souvenirs, installe une structure complexe qui mêle une temporalité et des niveaux narratifs hétérogènes. L'activité mémorielle se manifeste par la présence d'un je intermédiaire qui rappelle sa vie passée. En ravivant ce qui a été vécu par le truchement du souvenir, le locuteur entend montrer sa véritable personne mais la scénographie des Confessions est le fruit d'un dispositif énonciatif qui suppose deux subjectivités - celle du moi raconté et celle du narrateur - et qui ne laisse jamais le récit du je intermédiaire s'échapper de l'emprise du locuteur. Les marques de sa présence sont plus ou moins explicites dans les différents types de narration. Par exemple, la présence du narrateur peut se manifester à côté du je intermédiaire sans « rupture » nette entre deux modalités narratives, dans un même énoncé :

Tel est le narré fidèle de ma demeure à l'Ermitage, et des raisons qui m'en ont fait sortir. Je n'ai pu couper ce récit, et il importait de le suivre avec la plus grande exactitude, cette époque de ma vie ayant eu sur la suite une influence qui s'étendra jusqu'à mon dernier jour. ${ }^{14}$

A la fonction métanarrative assumée par le je intermédiaire («cette époque de ma vie », « ayant eu sur la suite », «jusqu'à mon dernier jour ») s'ajoutent la fonction de régie (« tel est le narré», «je n'ai pu couper ce récit ») et la fonction de communication ( « fidèle », « il importait », « suivre avec exactitude ») qui relèvent du locuteur. Celui-ci commente son travail d'écriture en établissant une relation intime entre les faits vécus par le je narré, l'interprétation qui leur donne du sens, les choix narratifs et l'adresse au lecteur.

La représentation de Jean-Jacques que le locuteur construit «comme un autre » peut mener à penser le je narré comme un personnage et la réalité autographique risque d'être ébranlée. Les fragments au présent de généralisation introduisent une dimension extra-temporelle dans le déroulement chronologique de la narration, la modalisation, la généralisation, l'expression de l'omniscience et du jugement du locuteur, les bilans et les prolepses, l'intrusion d'énonciateurs à la première personne dans le cadre de lettres insérées par le locuteur donnent le sentiment d'un univers créé, d'une double appartenance autographique et romanesque.

A mesure que le temps raconté se rapproche du temps de l'écriture, le souvenir devenu proche change de fonction: il est une adresse à un public de lecteurs contemporains qui partagent le même univers et connaissent les événements évoqués. Autant le je de mémoire est un moi d'expérience, sans visée argumentative, autant le locuteur, lui, poursuit l'objectif de témoigner, d'exposer des faits, des portraits en actes, des rencontres. La pseudo gratuité narrative de la première partie des Confessions disparait dans la 
deuxième partie au bénéfice du témoignage nécessaire pour échapper à un complot. La monstration du héros-narrateur devient démonstration du locuteur : on est passé à une autre « scène ». Les Confessions ne sont plus le portrait de son auteur, elles sont envahies par la relation pure et simple des rapports entre Rousseau et ses contemporains pris à témoin, comme s'il s'agissait des minutes d'un procès :

[...] ce fut Madame la marquise de Créqui, nièce de M. le bailli de Froulay, ambassadeur de Malte, dont le frère avait précédé M. de Montaigu dans l'ambassade de Venise, et que j'avais été voir à mon retour de ce pays-là. Madame de Créqui m'écrivit ; j'allai chez elle : elle me prit en amitié. J'y dînais quelquefois, j'y vis plusieurs gens de lettres, et entre autres M. Saurin, l'auteur de Spartacus, de Barneveldt, etc., devenu depuis lors mon très cruel ennemi sans que $\mathrm{j}$ 'en puisse imaginer d'autre cause, sinon que je porte le nom d'un homme que son père a bien vilainement persécuté. ${ }^{15}$

Les aventures du je narré-héros constituent l'argument, sans que la médiation d'un je intermédiaire soit nécessaire. Le caractère narratif, c'est-à-dire la textualité, le lien entre faits et sens, disparaissant, disparaît avec lui le sens donné à la vie de l'auteur et l'inscription du titre Confessions paraît plus appropriée. L'auteur semble «s'abîmer » dans le jeu social, et seul subsiste le commentaire rageur de la faillite de ses relations dans une sorte de procès verbal ${ }^{16}$.

Des Confessions aux Rêveries, l'omniprésence du locuteur derrière le je intermédiaire ne se manifeste pas de la même façon. Dans les Confessions (première partie), le je narré est tenu à distance par le découpage de l'exemplum et le procédé de la métalepse qui lui confèrent un caractère de détachabilité. Dans les Rêveries, la difficulté est surmontée par un nouveau procédé narratif d'enchâssement. L'introduction d'un promeneur de second niveau permet de parler de l'expérience d'aujourd'hui à travers celle d'hier, le locuteur prétendant appliquer sa mémoire à faire revivre les instants de rêverie créative dont son esprit ne serait plus capable.

[...] pour me contempler moi-même avant mon déclin, il faut que je remonte au moins de quelques années au temps où $[. .$.$] je m'accoutumais peu à peu à le nourrir de sa$ propre substance et à chercher toute sa pâture au-dedans de moi. ${ }^{17}$

Le présent du promeneur 1 est comme envahi par un autre présent, celui du promeneur 2 . Il va jusqu'à prétendre que le passé serait plus vivant que son propre présent. En faisant de son discours l'aboutissement de la réflexion du je narré, le locuteur manifeste que tout converge vers l'actualité de son propre commentaire. Cet effet de bouclage donne rétrospectivement à l'ensemble l'allure d'un récit tout entier au service de la réflexion philosophique. La rétrospective enchâssée est conforme au sens que Rousseau veut donner aux Rêveries comme une aventure intellectuelle : suite d'illustrations de la sagesse, et non suite chronologique d'événements. Ouvrant encore l'éventail des instances énonciatives qui font des Rêveries une œuvre polyphonique toute entière à la première personne, l'ego philosophique s'ajoute au je narré, au je intermédiaire et au je du locuteur.

Dans les Rêveries, le principe de l'itération est fondateur. En donnant le point de vue du promeneur 2, la réitération du ressentiment devient possible mais elle ne répète pas l'état de souffrance. Le texte veut précisément montrer que le point de vue du promeneur 1 n'est plus le même, parce qu'il aurait su trouver une issue à son malheur en se détachant des hommes. L'indécision dans laquelle est plongé le lecteur quant au repérage des strates temporelles et des instances énonciatives l'amène à renoncer à les distinguer pour se laisser transporter par le flux du texte comme le personnage de Rousseau-philosophe se coule dans le flux incessant de la vie :

Tout est dans un flux continuel sur la terre. Rien n'y garde une forme constante et arrêtée, et nos affections qui s'attachent aux choses extérieures passent et changent nécessairement comme elles. ${ }^{18}$ 


\subsection{Une théâtralisation du drame}

La stratégie communicationnelle des Dialogues est fondée sur la mise en abyme, qui décuple les effets du message aux lecteurs : portés par la double énonciation propre au discours théâtral et à la forme du dialogue, l'actualisation de la parole de l'auteur, y est toujours différée et médiatisée. Les émanations des je, marquées par la temporalité dans le premier écrit autographique, trouvent une inscription spatiale dans le second, Dialogues. Les actes de langage qu'accomplissait l'auteur des Confessions y sont délégués à des personnages.

J'ai pris la liberté de reprendre dans ces entretiens mon nom de famille que le public à juger à propos de m'ôter, et je me suis désigné en tiers à son exemple par celui de baptême auquel il lui a plu de me réduire. En prenant un Français pour mon autre interlocuteur, je n'ai rien fait que d'honnête et d'obligeant pour le nom qu'il porte ; puisque je me suis abstenu de le rendre complice d'une conduite que je désapprouve, et je n'aurais rien fait d'injuste en lui donnant ici le personnage que toute sa nation s'empresse de faire à mon égard. ${ }^{19}$

Le titre des Dialogues, Rousseau juge de Jean-Jacques pose déjà des actes : l'acte de dialoguer et celui de juger accroissant la force illocutoire de l'un comme de l'autre. Les Dialogues proposent une fiction comme condition d'accomplissement d'un procès. L'œuvre propose une défense qui se déroule en trois temps : le premier est celui de l'opposition entre les personnages (mode éristique), le second celui des expériences de la lecture et de la visite à J.-J., le troisième celui des témoignages qui rallient les opposants au même sentiment (mode heuristique). A l'issue des trois dialogues, se seront mêlées, dans un panorama exemplaire, les voix d'un lecteur acquis d'entrée de jeu («Rousseau » dans le premier dialogue), d'un lectorat désigné comme incompétent (dans les trois dialogues) et d'un lecteur conquis (le Français dans le troisième dialogue). L'investigation que mène chacun des personnages aboutit à une redistribution des rôles : les opposants cessent de l'être, la partie adverse n'est plus convoquée que par la citation. Le personnage de «Rousseau» et le Français chantent à l'unisson l'éloge de l'auteur, s'allient pour sa défense et participent de conserve à sa réhabilitation. La preuve est ainsi fournie qu'un retournement de situation est possible.

L'auteur fait œuvre de démiurge radical : en convoquant une figure incarnée dans un personnage et façonnée à sa convenance, il invoque également un colocuteur virtuel. Le Français est le témoin coopératif que Jean-Jacques Rousseau réclamait dans les Confessions, destinataire que l'auteur appelle de ses vœux lorsqu'il distribue le tract «A tout Français aimant encore la justice et la vérité ». Il exprime cette volonté d'entendre et de comprendre dont Rousseau prétend ne pas bénéficier dans la réalité mais dont il forge l'illusion, condition sine qua non de la réussite de l'argumentation.

Le principe dialogique des autographies de Rousseau est caractérisé par un type particulier d'adresse au lecteur. D'une œuvre à l'autre, la représentation des publics évolue. Sous la forme d'une adresse directe dans les Confessions, Rousseau dit ce qu'il fait pour se défendre ; dans les Dialogues, on assiste à la mise à distance du colocuteur fictif, ses arguments étant repris dans une configuration nouvelle. La demande faite dans les Confessions d'être jugé par un tribunal divin, sorte de relève du tribunal des hommes, est reprise explicitement dans Rousseau juge de Jean-Jacques. En revanche, les Rêveries prétendent ne plus s'inscrire dans ce cadre ; elles marquent le renoncement à toute place parmi les hommes en dénonçant comme obsolète le choix initial dont les Dialogues et les Confessions sont le théâtre :

Sentant enfin tous mes efforts inutiles et me tourmentant à pure perte $\mathrm{j}$ 'ai pris le seul parti qui me restait à prendre, celui de me soumettre à ma destinée sans plus regimber contre la nécessité. J'ai trouvé dans cette résignation le dédommagement de tous mes maux par la tranquillité qu'elle me procure et qui ne pouvait s'allier avec le travail continuel d une résistance aussi pénible qu'infructueuse. ${ }^{20}$ 


\section{Modalités de prise en charge du discours rapporté}

\subsection{La citation comme principe dialogique structurel}

Dans les Confessions, la représentation de la personne de Rousseau est affectée à l'exemplum. Dans les Dialogues, elle est explicitement le fruit de la fiction du témoignage. L'investissement de la seconde autographie de Rousseau dans les classes génériques évoquées poursuit un objectif qui n'est pas celui du dialogue philosophique, ni celui du factum: les Dialogues laissent paraître avant tout un auteur manifestant sa volonté de maîtrise de la réception de ses œuvres.

Ils constituent un discours citatif d'une rare densité où les personnages sont réduits aux rôles de porteparole ou de commentateurs. La scénographie repose sur la diffraction des instances énonciatives et sur le réseau que constituent paroles rapportées et scènes validées à propos de l'auteur Jean-Jacques. Dans un jeu de multiples miroitements, un spectre énonciatif se déploie : énonciateurs multiples, temporalité qui couvre passé, présent de la fiction et futur, redoublement fictionnel par l'imaginaire des énonciateurs, démultiplication par le faux...

Pour faire émerger la polyphonie complexe de ce texte dialogal, il s'agit, comme dans l'étude des Confessions, de considérer les instances distinctes de l'énonciation mais aussi les phénomènes de prise en charge du discours rapporté. L'énonciateur distinct du locuteur est défini comme l'être de discours censé « prendre (ou non) en charge » un point de vue et, la notion de point de vue correspond à l'entité de (non) prise en charge ${ }^{21}$.

Les Dialogues manifestent explicitement la nécessité de prendre en compte la parole d'autrui : la représentation de soi et la promotion de l'œuvre passent par la représentation du point de vue de l'ennemi pour le discréditer. C'est aussi entrer en relation avec le lecteur à travers une adresse plus ou moins directe pour l'amener à prendre position et de façon plus générale pour l'intéresser à des anecdotes tirées de sa vie personnelle. Impliquer son destinataire dans ses discours, c'est rapporter les mensonges de l'ennemi dont l'objectif est de disqualifier Rousseau pour poser la question de l'autorité, pour en inverser l'action néfaste, les contredire systématiquement. La manifestation de non prise en charge est une réponse efficace à ses détracteurs ; elle devient l'instrument de la promotion de l'auteur.

\subsection{Autocitation et promotion de soi dans les Dialogues}

Chambres d'écho de la parole d'autrui mais surtout de la parole de Jean-Jacques Rousseau, les Dialogues s'avèrent être un véritable manifeste autocitatif. Des citations erronées ou faussées de Jean-Jacques Rousseau rapportées par la bouche d'un personnage (le Français) pour faire apparaître son erreur de perception et souligner son indigence :

\footnotetext{
Rousseau: «Vous ne lui attribuez donc plus ces autres livres qu'on publie journellement sous son nom, et dont on a soin de farcir les recueils de ses écrits pour qu'on ne puisse plus discerner les véritables ? ${ }^{22}$
}

A ces propos s'ajoutent ses propres paroles que Jean-Jacques Rousseau met en scène dans des énoncés prononcés par des personnages qui portent ses nom et prénom. Ne peuvent logiquement faire justice au philosophe retiré du monde que des témoins visuels comme le personnage « Rousseau » qui doit rapporter au Français des preuves de l'intégrité de J.-J. après une visite qu'il lui fait, ou les lecteurs de ses livres comme le Français, lequel doit rendre compte de sa lecture du philosophe et la confronter avec celle d'autres lecteurs. Les paroles de J.-J. ne sont, en toute cohérence, que des paroles rapportées puisque qu'il a choisi de se taire : il prétend que ses paroles sont plagiées ou trahies, par absence d'adhésion.

Les Dialogues exemplifient la distinction entre « locuteur » et « énonciateur » dans toute sa complexité : l'autocitation se manifeste par le truchement de deux énonciateurs, les écrits de «J.-J. » lus et rapportés par le Français comme ses écrits et ses paroles rapportés par «Rousseau », toutes renvoient à l'auteur Jean-Jacques Rousseau. Quel que soit l'énonciateur désigné, tous citent finalement le locuteur premier, 
devant lequel s'interpose la figure incarnée par le personnage de l'auteur exilé, J.-J. Le statut d'autocitation des paroles rapportées de J.-J. apparaît à diverses reprises sous les traits de :

- L'auteur signataire des notes en bas de page. J.-J. souligne ironiquement dans les Dialogues l'identité qu'il partage avec le personnage de l'Auteur. Elle se trouve renforcée, du fait du dédoublement des personnages supposé aider à « objectiver» le débat ${ }^{23}$. Accomplissant un projet de commentaire total, l'auteur intervient également par intrusion ironique pour «jouer sa partie » dans l'espace infrapaginal. Les notes en bas de page sont une adresse directe de Rousseau au lecteur. «Rousseau » évoque le texte que le lecteur a entre les mains :

Enfin malgré la résolution qu'il avait prise en arrivant à Paris, de ne plus s'occuper de ses malheurs ni reprendre la plume à ce sujet [...], il a écrit en forme de dialogue une espèce de jugement d'eux et de lui assez semblable à celui qui pourra résulter des nos entretiens. ${ }^{24}$

ou encore, il stigmatise le fait de se constituer comme l'avocat de soi-même :

$\underline{\text { Rousseau }}$ : Ce que j'ai vu ne sont que des choses bien communes en apparence mais très rares en effet. Ce sont des récits qui d'ailleurs conviendraient mal dans ma bouche, et pour les faire avec bienséance, il faudrait être un autre que moi. ${ }^{25}$

Comptez-vous pour rien le calcul des voix, quand vous êtes seul à voir autrement que tout le monde ? $^{26}$

- Un locuteur fictif, le personnage de «Rousseau », porte-parole de Jean-Jacques. En cela, il redouble la première délégation de parole de l'auteur Jean-Jacques Rousseau qui l'implique dans le dialogue et confirme la situation de communication inédite que présente le titre Rousseau juge de JeanJacques. Il est dans une position de totale adhésion, puisqu'il prend en charge la parole du philosophe pour le défendre auprès du Français. Dans le deuxième dialogue, il rapporte l'échange épistolaire et les conversations qu'il a avec J.-J. lors de sa visite. Il fait état, au discours direct, de la démonstration que fait J.-J. pour justifier ses choix de vie. «Rousseau » évoque même les paroles qui auraient pu être les siennes et celles du Français dans un irréel du passé où dans l'irréel du futur :

Il me semblait que sans trop nous compromettre nous aurions pu leur dire : «Nous ne saurions approuver qu'aux dépens de la justice et de la sûreté publique, vous fassiez à un scélérat une grâce tacite qu'il n'accepte point et qu'il dit n'être qu'une horrible barbarie que vous couvrez d'un beau nom. [...]. » En leur parlant ainsi nous les aurions forcés à s'expliquer ouvertement, ou à convenir tacitement de leur imposture $[\ldots]^{27}$

- Un second locuteur fictif, le personnage du Français, lequel passe de l'adhésion totale à la parole des ennemis de Rousseau à l'adhésion totale à celle de J.-J. Une compilation de textes réellement écrits par Jean-Jacques Rousseau est présentée par le Français comme ceux de J.-J. Ces extraits d'énoncés numérotés et classés sont présentés dans les Dialogues dans leur intégralité. Une double sélection préside à une telle exposition : la première sélection est motivée par la volonté de l'ennemi, la seconde relève du choix de Jean-Jacques Rousseau de les transcrire sous une forme qui a trahi sa véritable pensée. En présentant ainsi des passages satiriques qu'il a réellement écrits, Jean-Jacques Rousseau semble se soumettre au jugement négatif de ses adversaires et il met le lecteur des Dialogues en situation de réagir lui aussi négativement; mais, dans le même temps, il stigmatise la lecture erratique et aléatoire de son œuvre qui en dénature la cohérence. Cette parodie inscrite dans la situation d'énonciation même sert de scène repoussoir, et s'inscrit dans une stratégie plus générale de défense de son image :

[...] je n'ai fait qu'extraire et transcrire fidèlement les passages. Vous jugerez vousmême des effets qu'ils ont dû produire et des qualifications que dut espérer leur auteur sitôt qu'on put l'en charger impunément. ${ }^{28}$

Mais le dispositif citationnel des Dialogues semble en contradiction avec l'enseignement au « Français », fondé sur la nécessité de n'accorder aucun crédit à la parole rapportée de l'auteur. Les Dialogues s'adressent indirectement au lecteur pour promouvoir une lecture qui prétend se passer des commentaires 
des autres, tout en les convoquant massivement. En outre, le travail de réhabilitation accompli par l'œuvre autographique est idéalement relayé à la fin par le projet des personnages de prendre en charge des « mémoires » qui seraient élaborés à partir des paroles du maître...

\begin{abstract}
Si nous nous unissons pour former avec lui une société sincère et sans fraude, une fois sûr de notre droiture et d'être estimé de nous, il nous ouvrira son cœur sans peine, et recevant dans les nôtres les épanchements auxquels il est naturellement si disposé, nous en pourrons tirer de quoi former de précieux mémoires dont d'autres générations sentiront la valeur, et qui du moins les mettront à portée de discuter contradictoirement des questions aujourd'hui décidées sur le seul rapport de ses ennemis. ${ }^{29}$
\end{abstract}

\title{
4.3 Autocitation et promotion de soi dans les Rêveries
}

L'étude de l'énonciation à travers les marques de présence du locuteur nous montre que Rousseau exploite dans les Rêveries les ressources des deux autographies précédentes. Elles tirent parti du procédé dramatique de la scission entre les deux personnages des Dialogues «Rousseau » et J.-J. : dans les Rêveries, ce sont les promeneurs 1 et 2 qui permettent l'interaction fictive. La spécificité énonciative des Rêveries peut être résumée en trois points : une configuration dialogique met en scène un co-énonciateur donné comme un autre soi-même : le promeneur solitaire est doublé d'un ego memorans distinct du locuteur. A la démultiplication des voix venues du passé, s'ajoute la démultiplication des énonciateurs par rapport auxquels Rousseau se positionne en prenant ou non en charge leur énoncé. La sentence qui oriente la réflexion émane en général du locuteur Rousseau ; elle est commentée et étayée par son expérience et ses commentaires. Il accomplit en cela le travail critique dévolu en général aux lecteurs et il semble livrer lui-même à la postérité les scènes validées sur lesquelles se construit sa légende. En présentant son œuvre comme la résultante de ses difficultés, il établit un lien fondateur entre l'écriture de soi et sa vie : son écriture ne peut pas échapper à l'autographie.

L'introduction de l'instance énonciative d'un sujet de mémoire et la forte prégnance de marques énonciatives du modèle de la disputatio sont les manifestations du dialogisme auquel Rousseau prétend être réduit dans le discours monologal des Rêveries. Alors que dans les Confessions, le lecteur voit se dérouler la narration d'une vie de façon chronologique, la construction des Rêveries est éclatée, chaque Promenade semblant offrir une nouvelle réflexion philosophique. Parfaitement clos sur eux-mêmes sur le plan formel comme sur le plan énonciatif, les fragments que constituent les Promenades exploitent la polyphonie caractéristique de la disputatio pour une mise en scène de la parole de l'auteur.

Le modèle scolastique lui permet à la fois de se mettre au centre du dispositif à travers l'autocitation, tout en maintenant la problématique philosophique de son rapport à l'autre dont l'expression énonciative est le jeu de question-réponse. La disputatio est un dispositif essentiellement polyphonique. Les trois étapes de l'exercice sont représentés dans les Promenades : une sentence soutient une thèse (c'est la lectio); elle est accompagnée de commentaires destinés à faire comprendre des œuvres de nature religieuse, philosophique ou scientifique (cette mise à l'épreuve du raisonnement par des arguments contraires est la quaestio), et une synthèse à l'aide du raisonnement formel pour trouver une solution (c'est la determinatio) ; l'anecdote confirme ou infirme une thèse qui la précède - en cela elle constitue un apport à l'argumentation :

Je deviens vieux en apprenant toujours. Solon répétait souvent ce vers dans sa vieillesse. Il a un sens dans lequel je pourrais le dire aussi dans la mienne ; mais c'est une bien triste science que celle que depuis vingt ans l'expérience m'a fait acquérir. ${ }^{30}$ [...] Ainsi retenu dans l'étroite sphère de mes anciennes connaissances je n'ai pas, comme Solon, le bonheur de pouvoir m'instruire chaque jour en vieillissant. ${ }^{31}$

La plupart du temps, Rousseau détourne les règles de la disputatio. Le déroulement canonique du raisonnement est bouleversé, rendant confuses l'identification de la sentence et la hiérarchie des auteurs et de leurs dires. 
Après lui avoir donné une forme dialogale dans les Dialogues, Rousseau intériorise le débat dans les Rêveries. Il se fait le locuteur unique d'un espace dialogique dont les énonciateurs pro et contra s'opposent à une troisième instance : l'autorité qui profère la sentence à discuter. Fondées sur la nécessité de l'étude de soi, les Rêveries prétendent réintroduire artificiellement la présence d'autrui. Le mode de la plainte et le constat de la perte que représente la solitude pour un être fondamentalement tourné vers les autres impose que la privation soit compensée. La nécessité du dialogue est prônée et contournée par des moyens énonciatifs et rhétoriques de deux ordres. Le recours à la réminiscence offre la possibilité de manifester un autre soi-même par l'intervention du passé. Le modèle de la disputatio montre une interaction verbale fictive. Comme son nom l'indique, il suppose la coénonciation et la controverse. Elles rendent compte de la recherche non pas d'un « temps perdu » mais d'un temps éternel à travers l'image d'un je qui transcende toute temporalité. Ce n'est plus la crédibilité de l'auteur qui est en jeu, mais le combat pour la gloire comme l'atteste le principe de l'autocitation, qui structurait déjà l'interaction dans les Dialogues.

\section{Conclusion}

A travers l'étude de l'énonciation dans les discours autographiques de Rousseau, nous avons fait émerger quelques constantes de l'autographie tout en prenant acte du fait qu'elle ne peut répondre à une catégorisation générique. Les titres de Confessions, de Dialogues, Rousseau juge de Jean-Jacques et de Rêveries sont justifiés par un positionnement dans l'interdiscours par lequel l'auteur prétend assurer sa défense. Une métascène judiciaire ${ }^{32}$ unit les œuvres en une sorte de vaste triptyque dont la visée serait la même et les formes multiples. Dans les trois œuvres, la métascène judiciaire que Rousseau organise se traduit par la simulation d'un procès, en écho à un autre procès, bien réel. Les discours autographiques de Rousseau prétendent agir en orientant la lecture à travers cette référence commune. Cette métascène revêt une fonction cardinale : elle constitue un premier lieu de rencontre du public et de l'œuvre.

L'étude des marques linguistiques de l'énonciation montre une certaine diversité dans la présentation de soi : l'usage de la première personne doit être envisagé comme l'expression de temporalités et/ou de perspectives illocutoires différentes et le dédoublement d'un «il » apparaît propre à rendre compte de l'apparente contradiction qui consiste à vouloir se faire connaître tout en prétendant se retirer du monde. La participation au monde passait par la défense et l'illustration de sa personne dans les Confessions et dans les Dialogues, dans les Rêveries, l'exil véritable s'accompagne du retrait de la scène judiciaire, la seule scène qu'il lui était possible d'occuper parmi les hommes. Elles complètent ainsi le dispositif de défense de Rousseau; la justification de son renoncement et la délégation à un ego memorans à peine distinct du locuteur sont une nouvelle confirmation de sa sincérité : il aurait tout dit.

Mais surtout, le principe autocitationnel qui préside aux deux dernières autographies conduit à penser que plus que l'expression de soi, c'est la volonté de laisser une œuvre qui s'affirme dans les écrits du philosophe. Le reconditionnement énonciatif des Confessions aux Rêveries manifeste la volonté de subsumer l'homme et l'œuvre passée sur le mode de la spéculation.

Au delà du corpus traité ici, la notion de reconfiguration discursive - en production comme en réception conduit à une réflexion plus générale sur le fait autographique et l'objectif paradoxal qu'elle poursuit : écrit métadiscursif, lieu de la construction de la figure d'auteur et de la glorification de son œuvre, l'autographie prétend à l'intransitivité propre aux textes fondateurs ${ }^{33}$. L'enjeu décisif qui préside à la prolifération des tentatives autographiques est fondé par le désir de l'artiste de faire passer un peu du corps dans le pur souffle de son verbe pour atteindre à l'immortalité de son nom par la grâce de son lecteur.

\section{Références bibliographiques}

Bourdieu P. (1986), «L'illusion biographique », Actes de la recherche en Sciences sociales, 62-63, p. 69-72.

Bres J. et Nowakowska A. (2006), «Dialogisme : du principe à la matérialité discursive ». Recherches linguistiques, 28 , p. 21-48. 
Charaudeau P. (1995), « Le dialogue dans un modèle de discours ». Cahiers de linguistique française, 17, p. 141-178.

Delormas, P. (2006). Les genres de la mise en scène de soi: les autographies de Rousseau. Thèse non publiée, Université de Paris 12.

- (2004). «Rousseau juge de Jean-Jacques : de la scène d'énonciation à la paratopie ». Champs du signe, 18, p. 3959.

Ducrot, O. (1984). Le dire et le dit. Paris : Minuit.

Genette, G. (1972). Figures III. Paris : Seuil.

- (1991). Fiction et diction. Paris : Seuil.

- (1979). Introduction à l'architexte. Paris : Seuil.

Grésillon, A. et Maingueneau, D. (1984). « Polyphonie, proverbe et détournement ». Langages, 73, p. 112-125.

Helkkula-lukkarinen, M. (1999). Construction de la scène d'énonciation dans A la recherche du temps perdu. Mémoires de la Société néophilologique de Helsinki, tome LVII.

Jaubert, A. (1990). La Lecture pragmatique. Paris : Hachette.

- (1996). «L'analyse pragmatique des textes littéraires ». Travaux du Cercle linguistique de Nice, 18, p. 3-15.

- (1987). Correspondance entre Henriette *** et J.-J. Rousseau. La subjectivité dans le discours. Genève-Paris : Slatkine-Champion.

Maingueneau, D. (2004). Le discours littéraire. Paratopie et scène d'énonciation. Paris : A. Colin.

- (2004). «Retour sur une catégorie : le genre ». Texte et discours : catégories pour l'analyse. Editions Universitaires de Dijon, p. 107-118.

- (1993). Le contexte de l'œuvre littéraire. Enonciation, écrivain, société. Paris : Dunod.

- (1991). L'analyse du discours, Introduction aux lectures de l'archive. Paris : Hachette.

Mathieu-Castellani, G. (1996). La scène judiciaire de l'autobiographie. Paris : PUF.

Perelman, C. (1988). L'empire rhétorique. Paris : Vrin.

Perrin, L. (éd.) (2006). Recherches linguistiques, 28.

Rabatel, A. (2006). "Autocitations et autobiographie dans Du sens, de Renaud Camus», Texte, 39-40, L'autobiographique, p. 81-118.

Ricœur, P. (1983). Temps et récit, I. Paris : Seuil.

Schaeffer, J.-M. (1986). «Du texte au genre. Notes sur la problématique générique ». Théorie des genres. Paris : Seuil.

${ }^{1}$. Voir, pour une justification détaillée de la création d'un tel néologisme, P. Delormas, Les genres de la mise en scène de soi, thèse non publiée, Paris 12, 2006.

2. P. Ricœur, Temps et récit, I, Paris, Seuil, 1983, p. 117.

3. Ibidem, p.118.

${ }^{4}$. Cf. A. Grésillon et D. Maingueneau, «Polyphonie, proverbe et détournement », Langages, 73, 1984, p. 112-125. On oppose «subversion» à « captation»; ce sont deux stratégies opposées de réinvestissement d'un genre de discours dans d'autres. Dans la subversion, l'imitation permet de disqualifier l'autorité du texte ou du genre source pour se légitimer.

5. Confessions, Livre I, p. 5.

${ }^{6}$. Cf. G. Pineau et J.-L. Le Grand, Les histoires de vie, Paris, PUF, coll. « Que sais-je ?», 1993. 
${ }^{7}$. Rappelons que les classes ainsi désignées sont des séries qui se construisent en référence à des textes antérieurs, proposés comme modèle reproductible.

${ }^{8}$. S. Maza en retrace l'histoire et les enjeux dans son ouvrage Vies privées, affaires publiques, les causes célèbres dans la France prérévolutionnaire, Paris, Fayard, 1997.

9. Dialogues, Premier Dialogue, p. 726.

${ }^{10}$. Dialogues, « Du sujet et de la forme de cet écrit », p. 663.

${ }^{11}$. Voir à ce sujet S. Guellouz, Le Dialogue, Paris, PUF, coll. « Littératures modernes », 1992.

${ }^{12}$. La primauté de l'entretien avec soi-même est soulignée dans F. de La Mothe Le Vayer, La Promenade, in Euvres complètes, Genève, Slatkine Reprints, tome IV, vol. 1 ( $1^{\text {ère }}$ éd. 1662 et 1663).

${ }^{13}$. Rêveries, Septième Promenade, p. 1062.

${ }^{14}$. Confessions, Livre IX, p. 488.

${ }^{15}$. Confessions, Livre VIII, p. 372.

${ }^{16}$. Les commentateurs ont en général attribué le changement de «ton» à des circonstances extérieures, à des désordres psychologiques. L'échec de l'autoportrait serait l'expression de la dissolution, du sentiment de perte de substance, du processus d'autodestruction dans lequel aurait sombré Rousseau.

${ }^{17}$. Rêveries, Deuxième Promenade, p. 1002.

18. Rêveries, Cinquième Promenade, p. 1046.

${ }^{19}$. Dialogues, p. 663.

${ }^{20}$. Rêveries, Première Promenade, p. 996.

${ }^{21}$. Cf. O. Ducrot, «Esquisse d'une théorie polyphonique de l'énonciation», Le dire et le dit, Paris, Minuit, 1984, chap. VIII, p. 171-233. Voir également L. Perrin (éd.), « Le sens et les voix. Dialogisme et polyphonie en langue et en discours », Recherches linguistiques, 28, 2006.

${ }^{22}$. Cf. Dialogues, Troisième Dialogue, p. 933.

${ }^{23}$. Le je narré est désigné explicitement comme «l'homme », à la troisième personne. Le procédé est annoncé par la Lettre à Hume: la scène épistolaire, de régime élocutif y bascule dans le régime délocutif par l'usage de la troisième personne grammaticale : le destinataire de la Lettre y devient un personnage, désigné comme témoin au procès que lui intente Rousseau. La vertu de ce déplacement est de décupler le spectre pragmatique du discours et de démultiplier ainsi son efficacité dans l'argumentation de l'innocence de l'auteur.

${ }^{24}$. Dialogues, Troisième Dialogue, p. 836.

${ }^{25}$. Dialogues, Premier Dialogue, p. 797. Mais à la question décisive que pose le Français à Rousseau personnage quant au crédit que l'on peut accorder à un jugement isolé, celui-ci répond qu 'il est le seul à juger par lui-même, et pour cause....

${ }^{26}$. Ibidem, p. 698.

27. Dialogues, Troisième Dialogue, p. 947-948.

${ }^{28}$. Ibidem, p. 917.

29. Ibidem, p. 974. Dans le Deuxième Dialogue, pour condamner l'attribution abusive à l'auteur de certains énoncés, «Rousseau» rapporte des énoncés produits par des faussaires. Nous sommes donc amenés à lire la contrefaçon de J.-J. dans les Dialogues que signe Jean-Jacques Rousseau. La mise en 
perspective du faux et du commentaire qui condamne son usage est une façon habile de redoubler également la citation, car elle donne accès par défaut à la lecture du texte originel. La dénonciation du faux appelle la promotion du juste. Le Français rapporte lui aussi des propos attribués à J.-J., mais faussés par une décontextualisation. Le Français rapporte des paroles lues dans les écrits de ses adversaires.

${ }^{30}$. Rêveries, Troisième Promenade, p. 1011, début.

${ }^{31}$ Ibidem, p. 1023, fin.

32. Voir à ce sujet G. Mathieu-Castellani, La scène judiciaire de l'autobiographie, Paris, PUF, 1996.

33: Au sens de D. Maingueneau (1991), le terme d'archive renvoie aux «archétextes» qui ne se reconnaissent d'origine qu'eux-mêmes. 